\title{
Análisis de la conexión arte-matemáticas en los libros de texto de Educación Primaria
}

\section{Analysis of the art-mathematics conexion in elementary school textbooks}

\author{
Teresa F. Blanco ${ }^{1}$ \\ Valeria González-Roel ${ }^{2}$ \\ José-Manuel Diego-Mantecón ${ }^{3}$ \\ Zaira Ortiz-Laso ${ }^{4}$
}

\begin{abstract}
Resumen: En la educación STEAM el libro de texto sigue siendo un recurso presente en las aulas. Este artículo analiza la conexión arte-matemáticas en los libros de texto de Educación Primaria a través de las manifestaciones artísticas presentes en ellos. La muestra está formada por 24 libros de texto de Matemáticas y 24 de Educación Plástica de cuatro editoriales. Se identificaron 101 manifestaciones artísticas en Matemáticas y 123 en Plástica, clasificadas en cuatro categorías: Arquitectura, Obras pictóricas, Escultura y Mosaicos. El instrumento de análisis atiende a cuatro dimensiones que establecen la conexión arte-matemáticas: Ornamental, contextual, conceptual, y creativa. Los resultados revelan que, tanto en los libros de Matemáticas como en los de Plástica, la conexión ornamental prevalece sobre las demás, seguida de la contextual, conceptual y por último la creativa. A nivel general, la conexión
\end{abstract}

Fecha de recepción: 1 de mayo de 2020. Fecha de aceptación: 9 de junio de 2021.

1 Área de Didáctica de la Matemática, Facultad de Ciencias de la Educación, Universidad de Santiago de Compostela, teref.blanco@usc.es, orcid.org/0000-0003-4215-8677

2 Departamento de Didácticas Aplicadas, Universidad de Santiago de Compostela, valeria.roel@usc.es, orcid.org/0000-0002-0707-7604

3 Facultad de Ciencias, Universidad de Cantabria, josemanuel.diego@unican.es, orcid.org/0000-00024427-2724

${ }^{4}$ Facultad de Ciencias, Universidad de Cantabria, zaira.ortiz@unican.es, orcid.org/0000-0001-9629-2279 
arte-matemáticas es más rica en los libros de Plástica en todos los cursos de primaria. Como conclusión, se desprende la necesidad de adaptar los libros de texto a la educación STEAM, potenciando las conexiones conceptual y creativa de, y desde, ambas asignaturas.

Palabras clave: STEAM, Educación Plástica, Educación Primaria, libros de texto, matemáticas

\begin{abstract}
In STEAM education textbooks remain a common resource in classroom. This article analyses the art-mathematics connection in Elementary Education textbooks through the artistic manifestations identified in them. A sample of 24 Mathematics and 24 Visual Arts textbooks was taken from four publishers. 101 artistic manifestations in Mathematics and 123 in Visual Arts were identified and classified in four categories: Architecture, Pictorial Works, Sculpture, and Mosaics. The instrument of analysis comprises four dimensions establishing the art-mathematics connection: Ornamental, Contextual, Conceptual, and Creative. The results revealed that in both Mathematics and Visual Arts textbooks the ornamental connection prevails over the others, followed by the contextual, conceptual, and finally the creative one. In general, the art-mathematics connection is richer in the Visual Arts textbooks than in the Mathematics ones, for the entire primary education stage. In conclusion, we identify the need to adapt textbooks to STEAM education, strengthening the conceptual and creative connections of, and from, both subjects.
\end{abstract}

Keywords: STEAM, visual Arts, Elementary Education, textbooks, mathematics

\title{
1. INTRODUCCIÓN
}

Las tendencias educativas actuales promueven principalmente tres elementos clave del aprendizaje: la investigación basada en proyectos, el trabajo colaborativo y la integración de contenidos. Estos elementos se vienen fomentando de forma significativa a través de la educación STEM (en inglés, Science, Technology, Engineering y Mathematics) (Kim y Parck, 2012; Thibaut et al., 2018). En los últimos años se ha favorecido, además, la conexión entre las asignaturas STEM y las artes con el objetivo de potenciar la creatividad del estudiante (Conradty y 
Bogner, 2018; Consejo de la Unión Europea, 2018). De esta forma, las STEM han incorporado la A de Artes en lo que se conoce actualmente como educación STEAM (Colucci-Gray et al., 2019; McKeown, 2019; Quigley y Herro, 2016). El interés, la imaginación, la toma de decisiones, e incluso la capacidad para innovar son aspectos del individuo que se refuerzan cuando se trabajan las habilidades artísticas en relación con las asignaturas STEM (Clarke, 2019; Liao, 2016; Ludeña, 2019; Wynn y Harris, 2012). En ese sentido, Allina (2018) afirma que este tipo de prácticas promueven la creatividad y el interés por el arte, aumentan el nivel de participación del alumnado y pueden mejorar potencialmente su formacióndien las disciplinas STEM. Concretamente, al binomio arte-matemáticas se le atribuyen múltiples beneficios (Bequette y Bequette, 2012; Henriksen, 2017; Land, 2013; Liao, 2016). Portaankorva-Koivisto y Havinga (2019) destacan, por ejemplo, que las prácticas educativas que integran contenidos y destrezas de las áreas de Matemáticas y de Educación Artística despiertan el interés del estudiante por las expresiones culturales, favoreciendo discusiones matemáticas fructíferas que permiten consolidar conceptos y procesos matemáticos a priori abstractos.

La implementación de la educación STEAM depende de las políticas educativas impulsadas en cada país y se establece en los documentos curriculares oficiales. Requiere cambios en la formación de docentes, en el diseño de las prácticas educativas y en los recursos utilizados. Muchas de las investigaciones realizadas se centran en la formación del profesorado, otras en el diseño de prácticas STEAM, pero muy pocas analizan los recursos para adaptarlos a este nuevo enfoque educativo. En el caso de España, los libros de texto son el recurso preferente del profesorado a la hora de implementar en el aula lo que establece el currículo oficial (Area-Moreira y González, 2015; Santaolalla et al., 2015), y, por lo tanto, un primer indicador de los contenidos que se enseñan (Wang y Lu, 2018).

Teniendo en cuenta las consideraciones previas, el objetivo de este trabajo es analizar cómo se establece la conexión arte-matemáticas en los libros de texto de toda la etapa de Educación Primaria (6-12 años) en España. En particular, se analizan los libros de texto de Educación Plástica y Matemáticas de cuatro de las editoriales más utilizadas en el país. Este análisis debe partir de la revisión de los documentos oficiales que marcan el currículo español y que recogen las recomendaciones europeas a nivel de educación STEAM. 


\section{ENFOQUES DE LA EDUCACIÓN STEAM. ARTE Y MATEMÁTICAS}

Según English (2016), la integración de la A de artes en las STEM se puede producir desde dos enfoques diferentes. El primero consiste en un enfoque simplista de la educación STEAM en el que las artes se incorporan a las disciplinas STEM para potenciar la parte creativa de estas, pero manteniendo cada uno sus respectivos conocimientos en compartimentos estancos. El segundo enfoque se apoya en una concepción de la educación STEAM transdiciplinaria, donde todas las disciplinas aportan conocimientos y procedimientos para encajar en un proyecto de manera entrelazada (Bequette y Bequette, 2012; Costantino, 2018; Guyotte et al., 2014; Henriksen, 2017; Liao, 2016; Peppler y Wohlwend, 2018). Este enfoque de aprendizaje se centra en el diseño, indagación e integración de contenidos con la finalidad de resolver problemas o analizar situaciones (Conradty y Bogner, 2018; Henriksen, 2017; Quigley et al., 2020). Según Henriksen (2017), la educación STEAM se debe pensar como un punto de encuentro de las disciplinas a través de los contextos, donde lo fundamental es el diseño y el análisis. Esto supone proporcionar a los profesores recursos para que puedan llevar a cabo su incorporación a este enfoque educativo, ya que los lentes unidisciplinarios pueden interferir en el camino de ver dónde se conectan las disciplinas o los contextos del mundo real.

En particular, la conexión arte-matemáticas desde este segundo enfoque debe abordarse a partir de cada una de las dos disciplinas. En el ámbito artístico predominan la belleza estética y la armonía de los elementos en comparación con todo lo referido a la matemática, es decir, esta última es utilizada como una herramienta a la hora de crear una composición con un fin artístico. Las matemáticas originan creaciones que pueden ser consideradas artísticas y que en realidad son consecuencia de meras nociones matemáticas, encontrándose lo artístico y lo bello en la propia matemática de forma espontánea (Diego-Mantecón et al., 2019). Además, aportan conocimientos y técnicas que el artista pone en práctica a la hora de crear su obra; la mayoría procedimientos de tipo geométrico y analítico, que resultan realmente provechosos y prácticos desde el punto de vista técnico. En este sentido, el arte hace hincapié en el pensamiento creativo y sintético para aumentar el interés del estudiante y el aprendizaje en matemáticas (Guyotte et al., 2014). Desde el punto de vista matemático; cuando observamos el trazo de una obra pictórica, por ejemplo, se puede distinguir entre línea recta y curva, así como estimar su pendiente, estableciendo conexiones entre las matemáticas y el arte a través de la geometría. Desde el punto de 
vista artístico se puede examinar, entre otros aspectos, el grosor de dicho trazo, el lugar que ocupa en el lienzo y la técnica con la que ha sido creado. Igualmente, se puede analizar cómo expresa estados de ánimo y sentimientos y cómo comunica ideas (Portaankorva-Koivisto y Havinga, 2019).

Las tendencias educativas actuales promueven la implementación de experiencias de aula en las que se evidencie la conexión arte-matemáticas. Prueba de ello es que cada vez con más frecuencia, en los centros educativos, se llevan a cabo intervenciones en las que se abordan contenidos curriculares de estas dos asignaturas (Colucci-Gray et al., 2019; Ferreira dos Santos y de Melo Teles, 2012; Land, 2013; Roel et al., 2019; Zhbanova, 2019). Desde el punto de vista educativo, la posibilidad de aplicar los contenidos de una asignatura a diferentes contextos facilita su comprensión, a la vez que enriquece el propio conocimiento. Además, el hecho de que los contextos sean variados ayuda a que el alumnado perciba la realidad de la actividad humana como un todo (Rodríguez-Taboada y García-Agra, 2019).

\section{DISPOSICIONES CURRICULARES Y LIBROS DE TEXTO}

Actualmente, las recomendaciones de la Unión Europea y de los Estados Unidos fomentan diversas iniciativas para reforzar la relación entre las asignaturas científicas y el arte, siguiendo una pedagogía fundamentada en la indagación y colaboración con diversos agentes sociales (Consejo de la Unión Europea, 2018; Diego-Mantecón et al., 2021; Peppler y Wohlwend, 2018; Rolling, 2016). Concretamente, se sugiere impulsar la adquisición de competencias en ciencia, tecnología, ingeniería y matemáticas, teniendo en cuenta su vínculo con las artes. Se considera que un proceso de aprendizaje efectivo es aquel que potencia el aprendizaje por competencias y este viene marcado por la transversalidad, el dinamismo y el carácter integral de los contenidos (Consejo de la Unión Europea, 2018).

A pesar de que el currículo de Educación Primaria en España define los contenidos, criterios de evaluación y estándares de aprendizaje evaluables de manera disciplinar, diferentes apartados de la Ley Orgánica para la Mejora de la Calidad Educativa (LOMCE) resaltan la importancia de esa enseñanza interdisciplinar (Ministerio de Educación, Cultura y Deporte, 2014) marcada por las recomendaciones anteriores. Las disposiciones curriculares establecen siete competencias clave, de las cuales las que más se ajustan a las asignaturas de Matemáticas y Educación Plástica son la competencia Matemática y 
competencias básicas en Ciencia y Tecnología y la competencia en Conciencia y Expresiones Culturales, respectivamente. La primera se potencia en los bloques de contenidos de todas las asignaturas de primaria, y la segunda en los tres últimos cursos. La asignatura de Matemáticas recoge principalmente la necesidad de aprender mediante la puesta en práctica de conocimientos y procedimientos matemáticos en contextos funcionales y variados, y la asignatura de Plástica establece una relación directa con las matemáticas especialmente en el bloque de Dibujo Geométrico (Ministerio de Educación, Cultura y Deporte, 2014).

Una de las funciones de los libros de texto es de servir de intermediario entre el currículo oficial y las prácticas de aula (Fan, 2013; Fan et al., 2013; Sayers et al., 2021). Este recurso es una herramienta didáctica recurrente en la mayoría de los países, reflejando los contenidos curriculares (Braga y Belver, 2016; Ibáñez-Ibáñez et al., 2019) y ayudando a "legitimar contenidos y formas específicas del conocimiento escolar" (Cantoral et al., 2015, p. 10). Algunos profesores cuando hablan de los contenidos de la asignatura se refieren directamente al libro de texto y no a los documentos legislativos, debido a la confianza que este recurso les transmite (Pro y Pro, 2011; Thompson y Senk, 2014). Esta situación provoca que los libros de texto puedan llegar a determinar la práctica docente más que la propia ley curricular, especialmente en la asignatura de matemáticas, donde la dependencia en el uso de esta herramienta es mayor que en otras asignaturas. Es por ello por lo que el análisis de los libros de texto de Matemáticas posee una gran tradición internacional desde los años ochenta (Fan, 2013; Fan et al., 2013). En concreto, una de las líneas de investigación que mayor interés ha suscitado es el análisis de los contenidos curriculares en distintos niveles educativos y contextos (Fan et al., 2013). Tanto es así que, como recogen Schubring y Fan (2018), esta línea se ha considerado recientemente en las revistas y congresos de educación matemática más prestigiosos.

En educación primaria, algunos estudios han examinado los libros de texto de Matemáticas de todos los cursos de la etapa de al menos una editorial (Mengual et al., 2017), mientras que otros han considerado los libros de texto de un único curso de dos o más editoriales (Aké y Godino, 2018; Sayers et al., 2021). Estas investigaciones han analizado contenidos matemáticos, identificando la ausencia de actividades que promuevan la adquisición de ciertas habilidades, así como diferencias entre las actividades propuestas entre editoriales (Aké y Godino, 2018; Mengual et al., 2017; Sayers et al., 2021). Desde una perspectiva no curricular, otros estudios se han centrado en analizar la función que desempeñan las ilustraciones -tablas, gráficos e imágenes- que comúnmente 
aparecen junto a los problemas (Dewolf et al., 2015). En el caso de la educación plástica, los estudios sobre libros de texto son mucho más escasos, como refieren Gómez-Pintado y Marcellán-Baraze (2017). Menor aún es el número de estudios que han ahondado en el contenido interdisciplinar de las matemáticas con otras disciplinas, en particular con el arte (Blanco et al., 2019a; Ferreira dos Santos y de Melo Teles, 2012).

\section{PREGUNTA DE INVESTIGACIÓN Y METODOLOGÍA}

Partiendo del enfoque transdisciplinar de las STEAM (Bequette y Bequette, 2012; Costantino, 2018; Guyotte et al., 2014; Henriksen, 2017; Liao, 2016), este estudio intenta responder a la pregunta de investigación: ¿¿De qué manera se establece la conexión arte-matemáticas en los libros de texto de cada una de estas disciplinas? La respuesta a esa pregunta requiere analizar esa conexión bajo una lente artística en los libros de texto de Matemáticas y una lente matemática en los libros de texto de Plástica.

\subsection{MUESTRA E INSTRUMENTO DE RECOGIDA DE DATOS}

Se ha utilizado una muestra intencionada, seleccionándose las cuatro editoriales con más difusión en España para el contexto de primaria: Anaya, Edelvives, Santillana y SM. Se analizaron los libros de texto de las asignaturas de Matemáticas y Educación Plástica, de los seis cursos correspondientes a la Educación Primaria (6-12 años), revisándose un total de 48 libros, 24 por asignatura, entre los años 2014-2016.

El instrumento de recogida de datos se construyó atendiendo a cinco indicadores para cada una de las manifestaciones artísticas encontradas en los libros de texto. El primer indicador recoge los datos de la manifestación artística (título de la obra, autor y año); el segundo, la categoría de manifestación artística (Arquitectura, Obras pictóricas, Mosaicos, y Escultura); el tercero, los datos del libro de texto (editorial, curso, página); el cuarto, el lugar del tema en el que aparece la manifestación (introducción, contenido, actividades, problemas, ampliación, refuerzo); el quinto, el tipo de representación de la manifestación (foto, dibujo o referencia textual) y, por último, el sexto indicador, incorpora el contenido matemático y/o de plástica implicado (conceptos, procedimientos, técnicas de diseño, material). 


\subsection{ANÁLISIS DE DATOS}

El instrumento de análisis de datos se tomó de Diego-Mantecón (2019) y se adaptó para evaluar la conexión arte-matemáticas en libros de texto de primaria, tanto de Matemáticas como de Plástica. Este instrumento establece la conexión arte-matemáticas a través de cuatro dimensiones que atienden a la función que realizan las manifestaciones artísticas en los textos: Ornamental, contextual, conceptual, y creativa. Estas dimensiones no han de interpretase como estructuras independientes; de manera que una actividad puede aludir a más de una dimensión. Se aplican a las manifestaciones artísticas que aparecen en imágenes, ilustraciones o referencias textuales, a lo largo de cada uno de los libros, tanto en el contenido como en las actividades propuestas. Se optó por un análisis mixto. El análisis cuantitativo, de corte descriptivo, proporciona el número de manifestaciones de cada categoría y su distribución atendiendo al curso, a la editorial y a las dimensiones para cada asignatura. El análisis cualitativo tiene como objetivo entender en profundidad la conexión arte-matemáticas presente en los libros de texto de cada asignatura a partir del instrumento seleccionado. El estudio respecto a la variable curso, permitirá detectar si existe algún patrón o secuencia en la distribución del tipo y número de manifestación artística a lo largo de la etapa educativa. Asímismo, mostrará si prevalece más una dimensión que otra dependiendo de la edad del alumnado.

A continuación, se describen las dimensiones de este instrumento para cada asignatura.

La dimensión ornamental en los libros de texto de Matemáticas se caracteriza por emplear las manifestaciones artísticas con un fin puramente ilustrativo, sin proporcionar una relación directa entre los conceptos matemáticos presentados y la manifestación artística que los acompaña. De forma similar, en los libros de Plástica esta dimensión alude a los objetos matemáticos que ejercen una finalidad decorativa y no proporcionan un significado complementario a la actividad o contenido artístico que se refiere. En esta dimensión la relación arte-matemáticas es por lo tanto inconexa, y se da, por ejemplo, en situaciones donde aparecen ilustraciones o fotografías en los márgenes de los libros que no tienen una relación directa con el contenido matemático que aparece a su lado.

La dimensión contextual en los libros de texto de Matemáticas se observa en aquellos contenidos o actividades donde el arte se emplea como referente para trabajar las matemáticas. El elemento artístico funciona como un complemento del contenido matemático, sin ser este estrictamente necesario para la 
realización de las actividades o compresión de los contenidos matemáticos. En este caso, los elementos o manifestaciones artísticas sirven únicamente como justificación para abordar un concepto o procedimiento matemático. Por ejemplo, cuando se adjunta una imagen con sus dimensiones como parte de una actividad en la que se pide realizar cálculos para reforzar conceptos como perímetro o área. La dimensión contextual en los libros de Plástica se observa cuando se hace referencia a objetos matemáticos para llevar a cabo una tarea artística. Al igual que en los libros de Matemáticas, la existencia del objeto matemático como ilustración no es necesaria para desarrollar la actividad. Se puede decir que este actúa como una 'excusa' para trabajar las propiedades artísticas.

La dimensión conceptual en los libros de texto de Matemáticas hace alusión al arte para asentar conceptos matemáticos. En ella, el objeto artístico funciona como ejemplo o como esquema para facilitar la comprensión del concepto o propiedad matemática que se quiere enseñar o reforzar. El objeto artístico está directamente relacionado con la actividad que se propone y se recurre a este para facilitar el desarrollo o comprensión de la tarea matemática. En los libros de Educación Plástica, al igual que en los de Matemáticas, se hace referencia al objeto matemático como contexto para asentar conceptos artísticos y al arte para asentar conceptos matemáticos. Es decir, las alusiones artísticas y matemáticas actúan como referencia una de otra para asentar conceptos y comprender su significado de una manera más eficaz.

Por último, tanto en los libros de texto de Matemáticas como en los de Plástica, la dimensión creativa consiste en crear arte a través de conceptos y procedimientos matemáticos o artísticos. Dentro de las actividades que aluden a esta dimensión, surgen composiciones artísticas poniendo en práctica tales conceptos y procedimientos.

\section{RESULTADOS}

A continuación, se presentan los resultados del análisis correspondiente a los libros de texto de Matemáticas y a los libros de texto de Educación Plástica en relación, en primer lugar, con las manifestaciones artísticas presentes y el peso que tienen en las diferentes editoriales $y$, en segundo lugar, con las cuatro dimensiones que determinan la conexión arte-matemáticas. 


\subsection{MANIFESTACIONES Y EDITORIALES}

Tabla 1. Porcentajes de las manifestaciones artísticas por curso y asignatura

\begin{tabular}{crrrrrrrr}
\hline & \multicolumn{2}{c}{ Arquitectura } & \multicolumn{2}{c}{ Obras pictóricas } & \multicolumn{2}{c}{ Mosaicos } & \multicolumn{2}{c}{ Escultura } \\
& Mat & Plast & Mat & Plast & Mat & Plast & Mat & Plast \\
\hline Primero & 6,0 & - & 1,5 & 11,5 & 8,3 & - & - & - \\
Segundo & 6,0 & - & 1,5 & 23,1 & 8,3 & 11,1 & - & 11,1 \\
Tercero & 13,6 & 18,5 & - & 1,4 & 8,3 & 11,1 & - & 38,8 \\
Cuarto & 51,5 & 14,8 & 1,2 & 15,9 & 33,3 & 33,3 & - & 27,7 \\
Quinto & 27,2 & 55,5 & 7,5 & 15,9 & 33,3 & 33,3 & - & 16,6 \\
Sexto & 34,8 & 18,5 & 68,7 & 18,8 & 8,3 & 11,1 & 1 & 16,6 \\
\hline
\end{tabular}

Se encontraron 101 manifestaciones artísticas en los 24 libros de texto de Matemáticas seleccionados. En la tabla 1 se recogen los porcentajes de las manifestaciones artísticas por curso y asignatura. La categoría que predomina en los libros de Matemáticas es Arquitectura con 66 manifestaciones, seguida por las Obras pictóricas con 16, a continuación, Mosaicos con 12 y, por último, Escultura con solo 7 manifestaciones. La categoría Arquitectura apareció en los libros de Matemáticas de todos los cursos, concentrando un porcentaje mayor de representaciones en los tres últimos cursos. La presencia de Obras pictóricas es muy baja y casi inexistente en tercer, cuarto y quinto curso (7.5 es casi inexistente), sin embargo, aumenta considerablemente en sexto (68,7\%). Por último, la categoría Escultura solo está presente en el sexto curso.

En cuanto a los 24 libros de texto de Plástica, se encontraron un total de 123 manifestaciones artísticas diferentes. La categoría que predomina es Obras pictóricas con 69 manifestaciones, seguida por Arquitectura con 27 y por Escultura con 18. Por último, solo se encontraron 9 manifestaciones para la categoría Mosaicos. La distribución por cursos no muestra ninguna manifestación en las categorías Arquitectura, Escultura y Mosaicos para primero (tabla 1). La cantidad de imágenes que aluden a pintura en los dos primeros cursos son muy similares y en cuarto y quinto el porcentaje es exactamente igual. Es en el último curso donde se encuentra el mayor número de manifestaciones en Obras pictóricas como se puede observar en la tabla 1. En cuanto a la categoría Mosaicos, predomina en los cursos de cuarto y quinto con un 33,3\% y el mayor porcentaje de manifestaciones para la categoría Escultura se obtiene en tercer curso (38,8\%). 
En cuanto al análisis de las editoriales (tabla 2), en los libros de Matemáticas la editorial Santillana es la que tiene una representación más alta de manifestaciones artísticas $(58,8 \%)$ y junto con SM $(28,3 \%)$ son las dos editoriales que tienen representación de todas las categorías. En estos libros, la editorial que tiene más peso en la categoría Arquitectura es SM (55\%). En los libros de texto de Plástica, la editorial Edelvives es la que tiene una representación más alta de manifestaciones artísticas (50,2\%), mientras que la editorial Anaya es la que tiene el porcentaje más bajo de manifestaciones (5,4\%). A pesar de ello, al igual que ocurre en todas las demás editoriales de los libros de Plástica, aparecen representaciones de todas las categorías.

Tabla 2. Porcentaje de manifestaciones artísticas por editoriales

\begin{tabular}{lrrrrrrrrrr}
\hline & \multicolumn{2}{c}{ Arquitectura } & \multicolumn{3}{c}{ Obras pictóricas } & \multicolumn{2}{c}{ Mosaicos } & \multicolumn{2}{c}{ Escultura } & \multicolumn{2}{c}{ Total } \\
& Mat & Plas & Mat & Plas & Mat & Plas & Mat & Plas & Mat & Plas \\
\hline SM & 55 & 35,7 & 18,8 & 6,3 & 25 & 11,1 & 14,3 & 33,3 & 28,3 & 21,6 \\
Anaya & 10 & 35,7 & 6,3 & 75,9 & 8,3 & 55,5 & - & 33,3 & 6,8 & 5,4 \\
Edelvives & 18,8 & 3,6 & - & 1,3 & 8,4 & 11,1 & - & 5,5 & 6,2 & 50,2 \\
Santillana & 16,25 & 25 & 75 & 16,5 & 58,3 & 22,2 & 85,7 & 27,8 & 58,8 & 22,9 \\
\hline
\end{tabular}

\subsection{DIMENSIONES}

A continuación, se presentan los resultados más relevantes en relación con las dimensiones que van a determinar el tipo de conexión arte-matemáticas en los libros de cada asignatura. La tabla 3 muestra el porcentaje de cada dimensión por manifestaciones artísticas y asignaturas.

Tabla 3. Porcentaje de dimensiones por manifestaciones artísticas y asignaturas

\begin{tabular}{crrrrrrrrrrr} 
& \multicolumn{1}{c}{} & \multicolumn{1}{c}{ Arquitectura } & \multicolumn{3}{c}{ Obras pictóricas } & \multicolumn{3}{c}{ Mosaicos } & \multicolumn{2}{c}{ Escultura } & \multicolumn{2}{c}{ Total } \\
\cline { 2 - 13 } & Mat & Plas & Mat & Plas & Mat & Plas & Mat & Plas & Mat & Plas \\
\hline Ornamental & 92,4 & 53,6 & 68,8 & 36,2 & 58,3 & 44,4 & 100 & 61,1 & 85,1 & 39,1 \\
Contextual & 13,6 & 32,1 & 31,2 & 52,2 & 25 & 11,1 & - & 33,3 & 16,8 & 36,9 \\
Conceptual & 4,6 & 21,4 & - & 5,8 & 25 & 22,2 & - & 11,1 & 5,9 & 9,9 \\
Creativa & - & 7,1 & - & 17,4 & - & 55,6 & - & 5,6 & 0 & 14,2 \\
\hline
\end{tabular}




\subsubsection{Libros de texto de Matemáticas}

La dimensión ornamental -manifestaciones artísticas con fin decorativo- apareció en una proporción elevada (85,1\%). Está presente en todas las manifestaciones artísticas con un porcentaje superior al 50\% (tabla 3). Como ejemplo de dimensión ornamental se muestra, en la figura 1 , el busto de Pitágoras sin referenciar. En el enunciado se menciona a dos matemáticos, Plutarco y Pitágoras, pero no se indica cuál de los dos es el que aparece en el busto. Estamos ante una manifestación artística escultórica cuyo fin es únicamente ilustrativo y no aporta ninguna información a la hora de desarrollar la actividad.

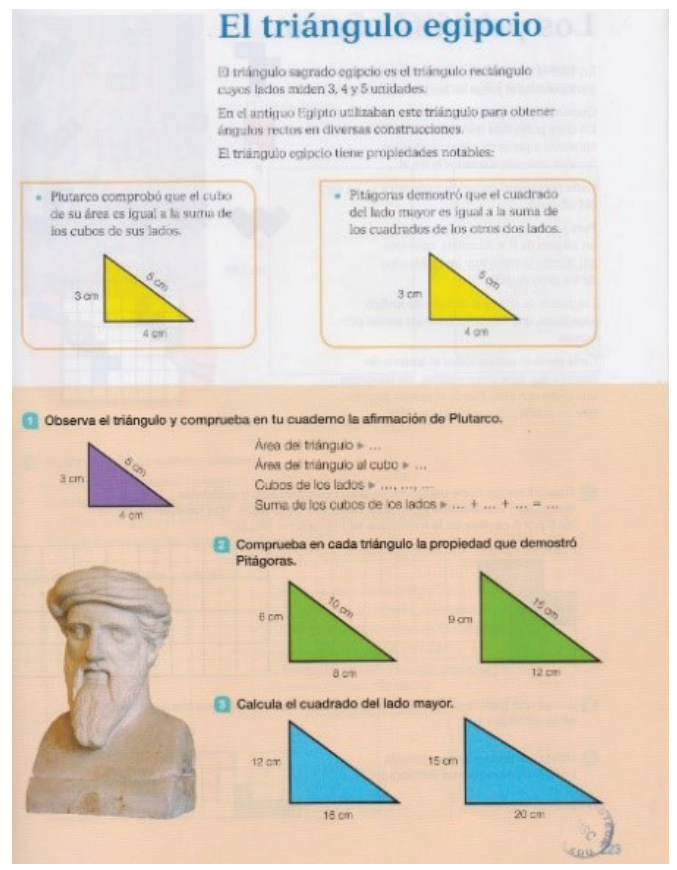

Figura 1. Dimensión ornamental: Busto de Pitágoras sin referencias (Almodóvar et al., 2015, p. 223).

La dimensión contextual -el arte actúa como referente para llevar a cabo la actividad propuesta- está presente en un 16,8\% de las manifestaciones artísticas, principalmente a través de Obras pictóricas. Un ejemplo para esta dimensión es 
el que se presenta en la figura 2. Los cuadros están correctamente referenciados, atribuyéndoles la misma función que podría desempeñar cualquier otro objeto rectangular, por ejemplo, el marco de una ventana. Las fotografías funcionan como contexto para realizar las actividades que debajo de ellas se demandan. Ambas imágenes actúan como contexto para realizar una serie de operaciones de cálculo relacionadas con las escalas de los cuadros que se muestran.

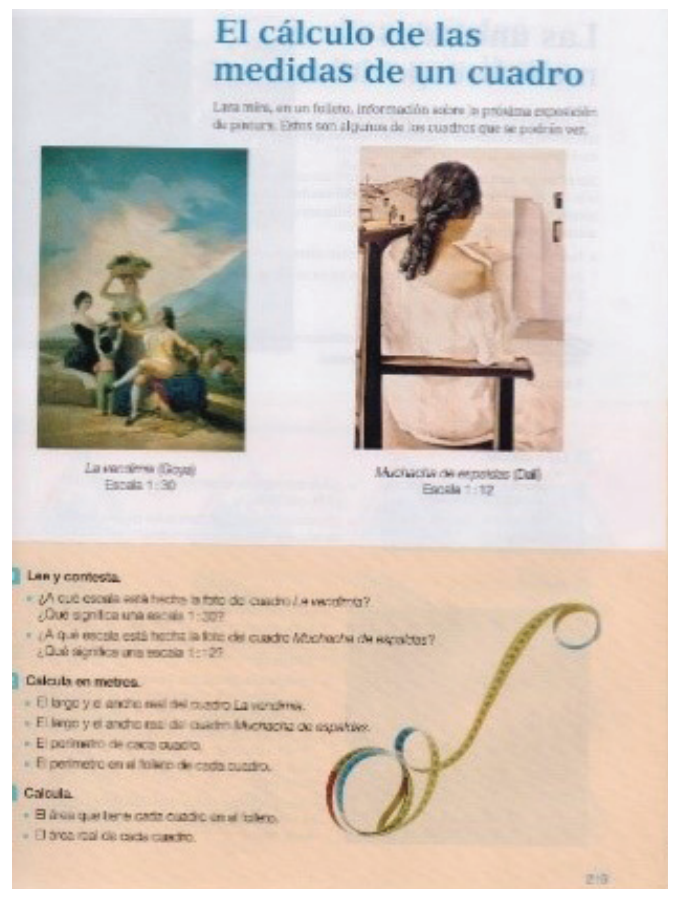

Figura 2. Dimensión contextual: La Vendimia (Goya) y Muchacha de Espaldas (Dalí) (Almodóvar et al., 2015, p. 219).

La dimensión conceptual, -arte como medio para asentar conceptos matemáticos- aparece en un 5,9\% y solo se encuentra en las manifestaciones arquitectónicas y en Mosaicos. En los libros de Matemáticas esta dimensión es inexistente en lo que a las Obras pictóricas y escultóricas se refiere. Como ejemplo para la dimensión conceptual, mostramos la actividad 1 de la figura 3, en la que se presenta un dibujo de las Torres KIO de Madrid. Aunque la obra es significativa en nuestro país y no ha sido referenciada en el texto, la actividad es importante 
desde el punto de vista matemático ya que requiere que se relacione una foto de una obra arquitectónica como un tipo de paralelogramo. Es decir, esta actividad implica una reflexión y puesta en práctica de conceptos matemáticos aprendidos.

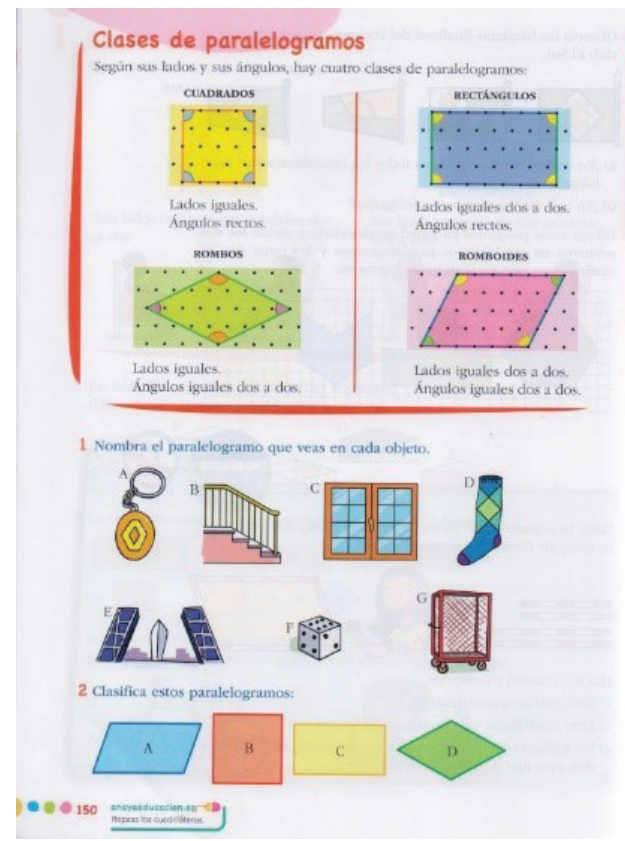

Figura 3. Dimensión conceptual: Torres KIO (Basanta y Fernández, 2015, p. 150).

Por último, la dimensión creativa -crear arte a través de las matemáticas- no aparece en ninguno de los libros de matemáticas analizados.

\subsubsection{Libros de texto de Educación Plástica}

La dimensión ornamental -donde los objetos matemáticos actúan con un fin decorativo- apareció en una proporción elevada (39,1\%) y en todas las manifestaciones artísticas con un porcentaje superior al 36\% en los libros de Plástica (tabla 3). Un ejemplo de esta conexión se puede ver en la actividad de la figura 4 donde se ilustran dos mosaicos de la Alhambra y se indica que están 
estructurados por formas geométricas. Sin embargo, no se profundiza en los conceptos matemáticos ni se propone ninguna actividad para poder trabajarlos.

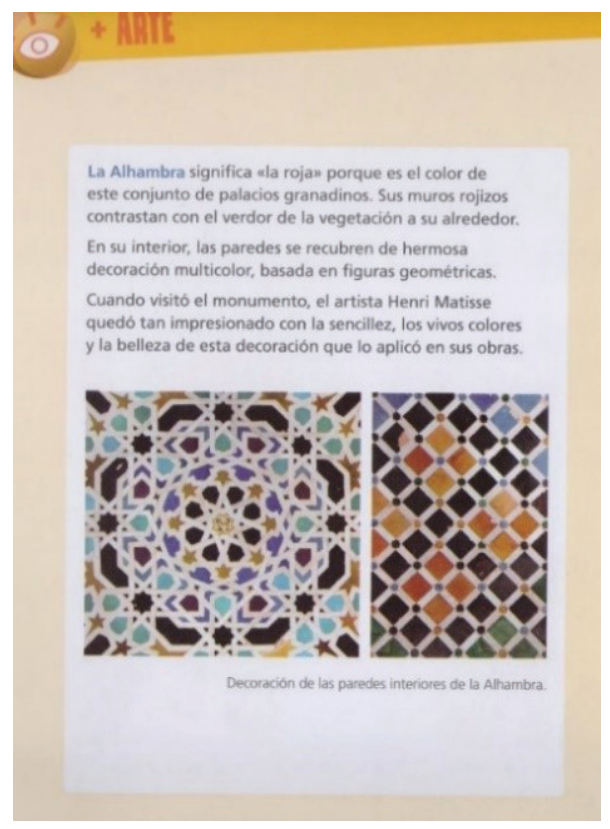

Figura 4. Dimensión ornamental: Mosaicos de la Alhambra (Baviera y Maiques, 2015a, p. 17).

La dimensión contextual -el concepto matemático actúa como referente para llevar a cabo la actividad propuesta- alcanza 36,9\%, siendo mayoritaria en la categoría Obras pictóricas. Como ejemplo se muestra la actividad de la figura 5. En ella se presenta la obra "Cisnes, Juncos y Lirios" de Crane de 1877, que se utiliza como contexto para completar un dibujo aplicando una simetría axial. La presencia de la obra en sí no es necesaria para construir la imagen simétrica de la parte que se presenta. 


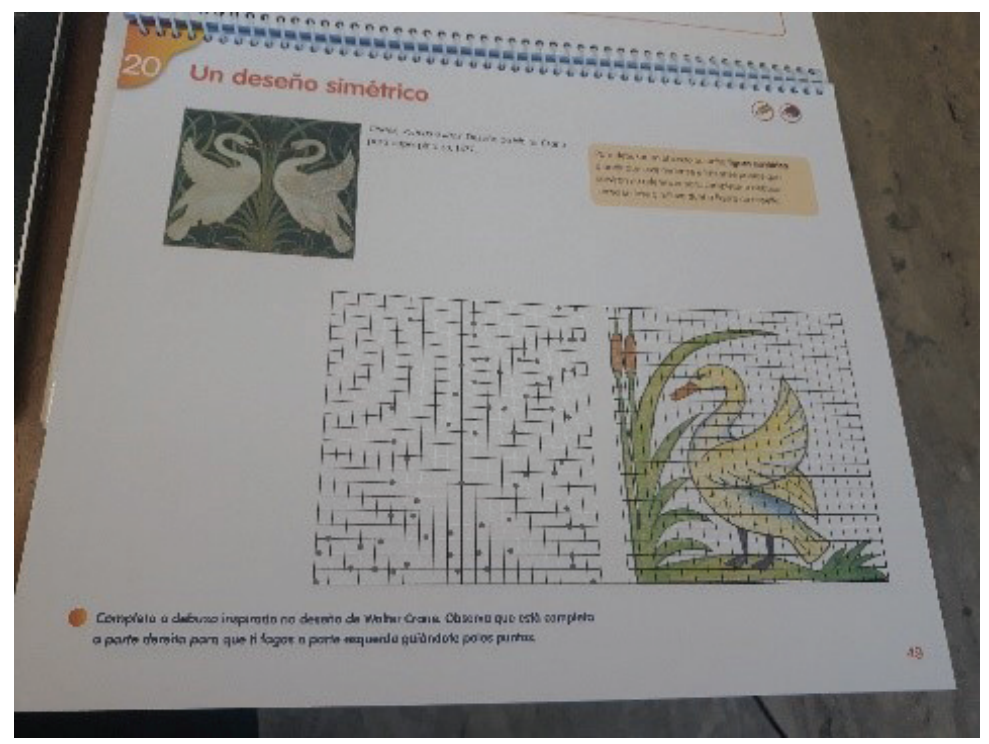

Figura 5. Dimensión contextual: Cisnes, Juncos y Lirios (Crane) (Herrero, 2014a, p. 49).

Por su parte, la dimensión conceptual -que hace referencia al arte como medio para asentar conceptos matemáticos- aparece en un 9,9\%. Esta dimensión se encuentra en todas las manifestaciones artísticas identificadas, y principalmente en las categorías Arquitectura y en Mosaicos con porcentajes del 21,4\% y $22,2 \%$, respectivamente (tabla 3 ). Los contenidos matemáticos que se trabajan a través de estas manifestaciones son habitualmente geométricos. Como ejemplo de esta dimensión se muestra la figura 6 , en la que se explica la construcción de la mediatriz de un segmento sin una alusión artística concreta. 


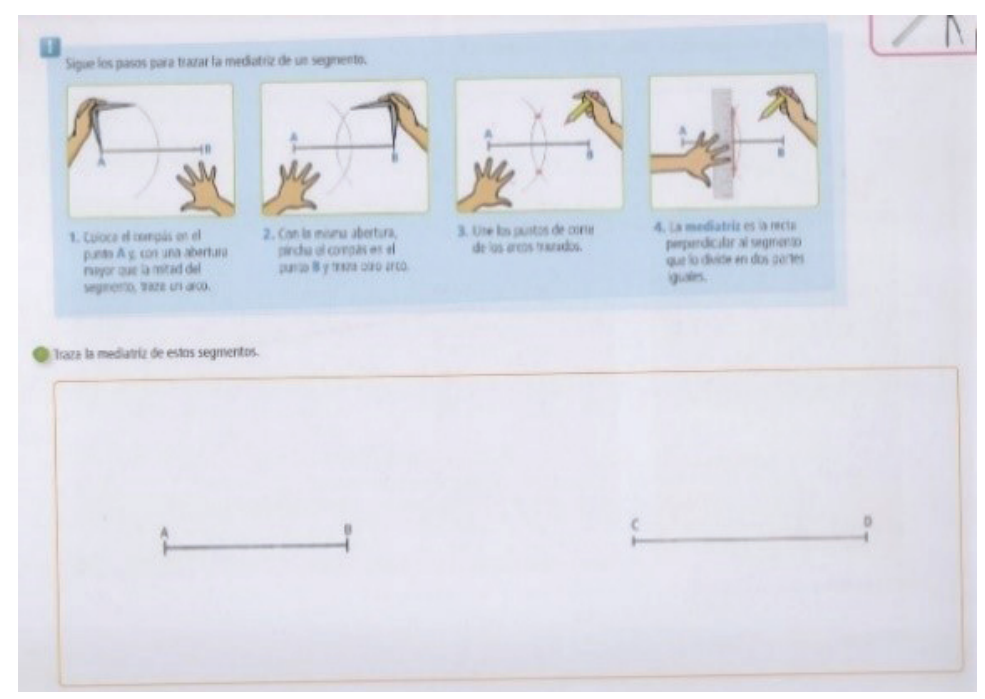

Figura 6. Dimensión conceptual: Mediatriz de un segmento (Baviera y Maiques, 2015b, p. 6).

Por último, la dimensión creativa -crear arte a través de las matemáticas- se detectó únicamente en un 14,2\%, y principalmente en la categoría Mosaicos con un 55,6\% (tabla 3). Se identificaron dos tipos de actividades: las relacionadas con crear composiciones artísticas y las relacionadas con reproducir Obras pictóricas de referencia mediante la utilización y puesta en práctica de conceptos geométricos. En la figura 7 se muestra un ejemplo de actividad en la que se pide al alumno crear un polígono estrellado y decorarlo. Inicialmente se refuerzan conceptos de naturaleza geométrica y se proporcionan los pasos necesarios para construir el polígono y la estrella del polígono, trabajándose de esta forma la dimensión conceptual y la creativa. 


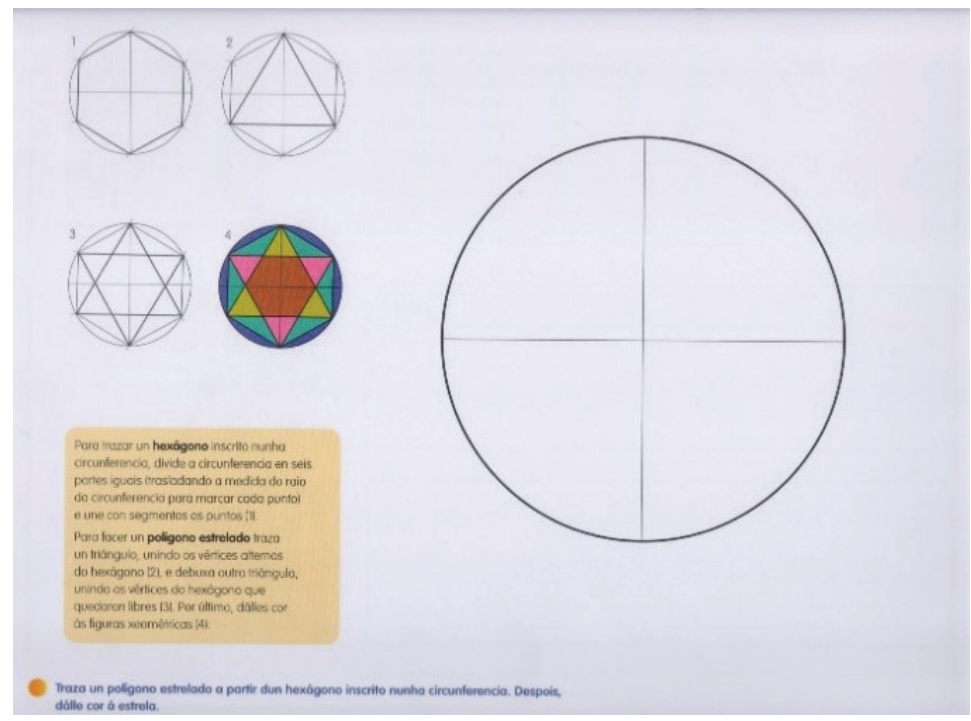

Figura 7. Dimensión creativa: Polígono estrellado (Herrero, 2014b, p. 35).

\section{DISCUSIÓN}

El análisis de manifestaciones artísticas por curso y asignatura mostró que el porcentaje total de manifestaciones es casi similar en las dos asignaturas. Globalmente, el curso que cuenta con los porcentajes más altos de manifestaciones artísticas es sexto en los libros de texto de Matemáticas, y quinto en los de Plástica (tabla 3). Si se comparan los libros de las dos asignaturas, vemos que en los de Matemáticas se produce un aumento de estas manifestaciones a medida que se avanza de nivel, mientras que en los de Plástica se produce un aumento repentino en quinto curso, descendiendo considerablemente en sexto. En ambas asignaturas, la presencia de los Mosaicos predomina en los cursos de cuarto y quinto con 33,3\%. En este caso, estos resultados son coherentes con el nivel de dificultad de los conceptos matemáticos (giro, traslación y simetría) y técnicas implicadas en el proceso de construcción. En general, los resultados obtenidos para los libros de Matemáticas se aproximan a los del análisis realizado por Diego-Mantecón et al. (2019) para secundaria, siendo la categoría 
Arquitectura la más presente en las manifestaciones artísticas encontradas. Sin embargo, la investigación de Ferreira dos Santos y de Melo Teles (2012), realizada con libros de texto de primaria y centrada en el concepto simetría, recoge un porcentaje bajo (8\%) para las actividades que incluían la categoría Arquitectura. En el caso de los libros de Plástica la manifestación más presente son las Obras pictóricas, lo que concuerda con la frecuencia de esta manifestación como tema en investigaciones y propuestas didácticas encontradas en la literatura. Si se presta atención a las editoriales, Santillana y Edelvives son las dos que muestran mayor representación de manifestaciones artísticas, la primera con un 58,8\% en los libros de Matemáticas y, la segunda, con un 50,1\% en Plástica (tabla 2). En cualquier caso, el número de manifestaciones artísticas, concordando con Sayers et al. (2021), no es un indicativo de la calidad de las tareas propuestas en torno a ellas, como se puede observar en la tabla 3.

El tipo de conexión arte-matemáticas que más se establece, tanto en los libros de Matemáticas como en los de Plástica es la ornamental (tabla 3), seguida de la contextual, la conceptual y, por último, la creativa que casi no tiene presencia en los libros de Plástica, y es inexistente en los de Matemáticas. El alto porcentaje de la conexión ornamental con respecto a las demás conexiones difiere del estudio de Dewolf et al. (2015) en el que se obtiene que las ilustraciones son esenciales para resolver las actividades. Por otra parte, estos resultados concuerdan con los obtenidos en Diego-Mantecón et al. (2019) para libros de texto de Matemáticas de secundaria, donde las diferencias más destacables las encontramos comparando las dimensiones conceptual y creativa. En cuanto a esta primera, en secundaria el porcentaje es del 23,9\% y, en primaria, se reduce hasta el 5,9\%. Finalmente, en la creativa, se observa que, aunque el porcentaje en primaria sea muy bajo (14,2\%), el referido a los libros de texto de secundaria es de 1,4\%.

Como afirma el Real Decreto 126/2014 (Ministerio de Educación, Cultura y Deporte, 2014), la asignatura de matemáticas pretende fomentar un enfoque globalizador e interdisciplinar de los contenidos. Por lo tanto, siguiendo las indicaciones del currículo, en la figura 1, ejemplo de conexión arte-matemáticas ornamental, se podría dar valor artístico a la escultura que aparece añadiendo, por ejemplo, información relativa a la época o al escultor que la realizó y a la biografía de Pitágoras y sus aportaciones en el ámbito matemático y así conectar contextualmente las disciplinas. En este caso se estaría dando prioridad a la indagación (Quigley et al., 2020) y potenciando la competencia en Conciencia y Expresiones Culturales. En la misma figura 1, también se podría trabajar el concepto triángulo egipcio de una manera artística y establecer una conexión 
conceptual. Para ello, se podrían identificar triángulos en Obras pictóricas y comprobar si estos son triángulos egipcios o, en su caso, si cumplen el teorema de Pitágoras. También, se podría pedir que realizasen composiciones artísticas utilizando solo estos triángulos cumpliendo algunas restricciones de material o de posición de los mismos en el plano, para trabajar la conexión creativa basada en el diseño (Henriksen, 2017). La actividad presentada en la figura 2 (conexión arte-matemáticas contextual) daría juego para realizar un trabajo de investigación, indagando sobre el uso de la escala en el arte. Asímismo, se podría solicitar la búsqueda de más obras de Goya y de Dalí y realizar una descripción comparativa entre los elementos que las conforman (escala, figuras, espacio, profundidad) y así establecer una conexión conceptual. Por último, en la actividad de la figura 3, ejemplo de conexión conceptual, también se podría realizar un trabajo de indagación sobre el arquitecto de tal obra y dónde se encuentra, así como intentar reproducirlas con algún material manipulativo o tecnológico y fomentar la conexión creativa. De esta forma se estaría trabajando la competencia en Matemáticas, y competencias básicas en Ciencia y Tecnología.

En los libros de Plástica la mayoría de las manifestaciones artísticas que aparecen están correctamente referenciadas, situación que no ocurre en los libros de Matemáticas. Sin embargo, su relación con las matemáticas no se explicita claramente, lo que implica una difícil comprensión del concepto matemático. Por ejemplo, en la actividad de la figura 4, se podría haber propuesto una actividad en la que el alumnado realizara una búsqueda sobre el origen de los mosaicos, profundizando en sus particularidades culturales, así como matemáticas (conexión contextual y conceptual). Asimismo, el alumnado desarrollaría la competencia en Conciencia y Expresiones Culturales al reconocer la calidad artística y el valor patrimonial de estas manifestaciones artísticas. Además, se podría establecer la conexión creativa solicitando al estudiante diseñar sus propios mosaicos explicitando sus conocimientos matemáticos sobre las transformaciones geométricas utilizadas e incorporando la tecnología para hacer los diseños a través de programas como GeoGebra o algunos específicos de diseño, incluso creando las piezas con la impresora 3D. Esta vinculación de la tecnología con el pensamiento creativo y el diseño artístico es un factor clave de la educación STEAM (Peppler y Wohlwend, 2018; Wynn y Harris, 2012). Esta propuesta concuerda con uno de los objetivos generales del currículo (Ministerio de Educación, Cultura y Deporte, 2014), relacionado con la creación de representaciones visuales y favorece la competencia en Matemáticas y competencias básicas en Ciencia y Tecnología. 
Es frecuente que en los libros de Plástica se utilice un concepto matemático para crear una producción artística. Es el caso del ejemplo de la figura 5, que solicita completar una simetría de eje vertical para obtener un cisne. Desde el punto de vista de la matemática y apoyando la investigación de Ferreira dos Santos y de Melo Teles (2012), no se proponen otros ejes de simetría como el oblicuo o el horizontal, ni tampoco se profundiza en el concepto utilizado. Desde el punto de vista de la educación artística, el espacio utilizado para el arte es uno de los pocos a nivel escolar donde se fomentan resultados divergentes y se valoran soluciones de diseño innovadoras (Rolling, 2016). En ese sentido, se podría trabajar con otros soportes y materiales diferentes a los habituales. Considerando lo que dicen Pro y Pro (2011) y Peppler y Wohlwend (2018) sobre expandir el potencial creativo a través de la tecnología en educación, se podrían reforzar, tanto la actividad de la figura 6 como la de la figura 7, utilizando, por ejemplo, la técnica lightpainting (Roel et al., 2019). De esta manera se estaría potenciando la conexión creativa de las dos asignaturas.

\section{CONCLUSIONES}

Este trabajo analiza la conexión arte-matemáticas, en libros de Matemáticas y de Plástica, de cuatro editoriales españolas a lo largo de toda la etapa de Educación Primaria. Si se presta atención a las editoriales, Santillana y Edelvives son las dos que muestran mayor representación de manifestaciones artísticas. En concreto, las categorías Arquitectura y Obras pictóricas son las más frecuentes tanto en los libros de Matemáticas como de Plástica. El bajo porcentaje de la categoría Escultura en los libros de Matemáticas y de la de Mosaicos en los de Plástica apunta hacia la necesidad de incorporar más actividades con representación de esas manifestaciones en las cuatro conexiones arte-matemáticas, ampliando las relaciones que se establecen entre las dos asignaturas y promoviendo la innovación.

El análisis de resultados ha mostrado que, globalmente, no se ha encontrado ningún patrón en la distribución de las manifestaciones a lo largo de la etapa educativa en ninguna editorial ni en ninguna de las asignaturas. Se puede decir que en los libros de Matemáticas hay una tendencia creciente hacia el número de manifestaciones representadas a medida que se avanza de curso. En los libros de Plástica esa tendencia se mantiene hasta quinto curso, produciéndose un descenso del 50\% en sexto, que este estudio no puede explicar. 
La conexión entre arte y matemáticas se establece a partir de cuatro dimensiones: ornamental, contextual, conceptual y creativa que son las que determinan el tipo de conexión arte-matemáticas. Los análisis evidenciaron que la mayoría de las manifestaciones artísticas identificadas tienen, tanto en los libros de Matemáticas como en los de Plástica, una función puramente ornamental. El porcentaje de la dimensión contextual fue mayor que el de la conceptual, lo que indica que las manifestaciones artísticas en los libros de ambas asignaturas se utilizan más para contextualizar actividades que como recurso para asentar conceptos matemáticos o artísticos. La conexión creativa se encontró en un porcentaje significativamente más bajo que el resto, incluso en los libros de Plástica, estando ausente en los libros de Matemáticas. Estos resultados, en los libros de texto de Plástica, resultan desconcertantes si tenemos en cuenta que la creatividad es uno de los aspectos más representativos de cualquier manifestación artística.

Es importante resaltar que, en los libros de Plástica, la conexión arte-matemáticas parece tener un papel más activo y equilibrado que en los libros de Matemáticas. Por un lado, se amplía parte del contenido artístico con conceptos y procedimientos matemáticos, lo que indica una mayor conexión conceptual entre las dos asignaturas que en los libros de Matemáticas. Por otro lado, en los libros de Plástica se encuentran representaciones de al menos tres de las cuatro conexiones en todos los cursos de la etapa educativa, con tendencia hacia las conexiones conceptual y creativa a medida que se avanza de curso.

En general, el estudio muestra que la conexión arte-matemáticas en los libros de texto de ambas asignaturas se guía por una perspectiva simplista de las STEAM, concentrando esa conexión en la ornamental y la contextual y alejándose de las recomendaciones curriculares que buscan promover el aprendizaje interdisciplinar entrelazando lo conceptual y lo creativo de cada disciplina. Se ha visto en la discusión que las actividades existentes se pueden reformular 0 ampliar para potenciar una conexión concreta. Esto conecta con la idea de que la potencialidad de los recursos y en particular, la de los libros de texto, viene dada por el uso que el docente hace de él (Thompson y Senk, 2014). De acuerdo con Allina (2018), hay que dar libertad a los profesores para que exploren el aprendizaje interdisciplinario, pero también hay que darle recursos que les ayuden a poder explorarlo. En ese sentido, en este trabajo se ha puesto de manifiesto que los libros de texto de Matemáticas y de Plástica no reflejan esas recomendaciones en materia de educación STEAM lo que sugiere la necesidad 
de diseñar libros de texto que potencien esa visión de la educación y, por tanto, la conexión conceptual y creativa de y desde ambas disciplinas.

Para finalizar, se debe tomar este estudio como un estudio exploratorio y con ciertas limitaciones. La primera de ellas hace referencia al libro de texto que, como recurso que es, depende del uso que haga el docente de él y, por tanto, la práctica puede variar de unos docentes a otros. Por otra parte, se ha analizado solo una parte de la educación artística ya que, como establece el Real Decreto 126/2014 (Ministerio de Educación, Cultura y Deporte, 2014), en España la Educación Artística comprende la Educación Plástica y la Educación Musical y en este estudio solamente se ha analizado la Educación Plástica. Por ese motivo, para completar esta investigación, sería necesario analizar también la parte correspondiente a la Educación Musical. Además, el estudio se podría ampliar con el análisis de libros de texto de otros países, incorporando así la variable cultural.

\section{AGRADECIMIENTOS}

Financiado por: FEDER/Ministerio de Ciencia, Innovación y Universidades Agencia Estatal de Investigación/_Proyecto EDU2017-84979-R.

\section{REFERENCIAS}

Aké, L. P., y Godino, J. D. (2018). Análisis de tareas de un libro de texto de primaria desde la perspectiva de los niveles de algebrización. Educación Matemática, 30(2), 171-201. https://doi.org/10.24844/em3002.07

Allina, B. (2018). The development of STEAM educational policy to promote student creativity and social empowerment. Arts Education Policy Review, 119(2), 77-87. https://doi.org/10.1080/10632913.2017.1296392

Almodóvar, J. A., García, P., Rodríguez, M., y Pérez, C. (2015). Matemáticas 6. Editorial Santillana.

Area-Moreira, M., y González, C. S. (2015). De la enseñanza con libros de texto al aprendizaje en espacios online gamificados. Educatio Siglo XXI, 33(3), 15-38. https://doi. org/10.6018/j/240791

Basanta, N., y Fernández, A. (2015). Matemáticas 3. Editorial Anaya.

Baviera, J., y Maiques, A. (2015a). Educación plástica 3. Editorial Luis Vives. 
Baviera, J., y Maiques, A. (2015b). Educación plástica 5. Editorial Luis Vives.

Bequette, J. W., y Bequette, M. B. (2012). A place for art and design education in the STEM conversation. Art Education, 65(2), 40-47. https://doi.org/10.1080/00043125.2012.115 19167

Blanco, T. F., González-Roel, V., y Diego-Mantecón, J. M. (2019a). Mathematics and Art in Primary Education Textbooks. En T. Jankvist, M. van den Heuvel-Panhuizen y M. Veldhuis (Eds.), Proceedings of the Eleventh Congress of the European Society for Research in Mathematics Education (CERME 11) (pp. 4737-4738). Utrecht, The Netherlands: Freduenthal Group y Freudenthal Institute.

Braga, G. M., y Belver, J. L. (2016). El análisis de libros de texto: una estrategia metodológica en la formación de los profesionales de la educación. Revista Complutense de Educación, 27(1), 199-218. https://doi.org/10.5209/rev_rced.2016.v27.n1.45688

Cantoral, R., Montiel, G., y Reyes-Gasperini, D. (2015). Análisis del discurso matemático escolar en los libros de texto, una mirada desde la Teoría Socioepistemológica. Avances de Investigación en Educación Matemática, 8, 9-28. https://doi.org/10.35763/ aiem.v1i8.123

Clarke, M. (2019). STEM to STEAM: Policy and practice. En A. de la Garza y C. Travis (Eds.), The STEAM revolution: Transdisciplinary approaches to science, technology, engineering, arts, humanities and mathematics (pp. 223-236). Springer. https://doi. org/10.1007/978-3-319-89818-6_15

Colucci-Gray, L., Burnard, P., Gray, D., y Cooke, C. (2019). A critical review of STEAM (science, technology, engineering, arts, and mathematics). Oxford research encyclopedia of education (pp. 1-26). Oxford University Press. https://doi.org/10.1093/acrefore $/ 9780190264093.013 .398$

Conradty, C., y Bogner, F. X. (2018). From STEM to STEAM: How to monitor creativity. Creativity Research Journal, 30(3), 233-240. https://doi.org/10.1080/10400419.2018.1 488195

Consejo de la Unión Europea (2018). Recomendación del consejo de 22 de mayo de 2018 relativa a las competencias clave para el aprendizaje permanente (2018/C 189/01). Diario Oficial de la Unión Europea, 4 de junio de 2018, 189, 1-13.

Costantino, T. (2018). STEAM by another name: Transdisciplinary practice in art and design education. Arts Education Policy Review, 119(2), 100-106. https://doi.org/10.1080/10 632913.2017 .1292973

Dewolf, T., Van Dooren, W., y Verschaffel, L. (2015). Mathematics word problems illustrated: An analysis of Flemish mathematics textbooks. Mediterranean Journal for Research in Mathematics Education, 14, 17-42. 
Diego-Mantecón, J. M., Blanco, T. F., Búa Ares, J. B., y González Sequeiros, P. (2019). Is the relationship between art and mathematics addressed thoroughly in Spanish secondary school textbooks? Journal of Mathematics and the Arts, 13(1-2), 25-47. https:// doi.org/10.1080/17513472.2018.1552068

Diego-Mantecón, J., Blanco, T., Ortiz-Laso, Z., y Lavicza, Z. (2021). STEAM projects with KIKS format for developing key competences. [Proyectos STEAM con formato KIKS para el desarrollo de competencias clave]. Comunicar, 66, 33-43. https://doi. org/10.3916/C66-2021-03

English, L. D. (2016). STEM education K-12: Perspectives on integration. International Journal of STEM Education, 3(3). https://doi.org/10.1186/s40594-016-0036-1

Fan, L. (2013). Textbook research as scientific research: Towards a common ground on issues and methods of research on mathematics textbooks. ZDM-The International Journal on Mathematics Education, 45(5), 765-777. https://doi.org/10.1007/s11858013-0530-6

Fan, L., Zhu, Y., y Miao, Z. (2013). Textbook research in mathematics education: Development status and directions. ZDM-The International Journal on Mathematics Education, 45(5), 633-646. https://doi.org/10.1007/s11858-013-0539-x

Ferreira dos Santos, L., y de Melo Teles, R. A. (2012). Pintar, dobrar, recortar e desenhar: o ensino da Simetria e Artes Visuais em livros didáticos de matemática para os anos iniciais do Ensino Fundamental. Bolema: Boletim de Educaçāo Matemática, 26(42A), 291-310. https://doi.org/10.1590/s0103-636×2012000100013

Gómez-Pintado, A., y Marcellán-Baraze, I. (2017). ¿Responden las imágenes utilizadas en educación plástica y visual a la propuesta curricular? Un análisis de las imágenes de los libros de texto de Educación Secundaria en la Comunidad Autónoma Vasca. Educación XX1, 20(1), 233-252. https://doi.org/10.5944/educxx1.17510

Guyotte, K. W., Sochacka, N. W., Costantino, T. E., Walther, J., y Kellam, N. N. (2014). STEAM as social practice: Cultivating creativity in transdisciplinary spaces. Art Education, 67(6), 12-19. https://doi.org/10.1080/00043125.2014.11519293

Henriksen, D. (2017). Creating STEAM with design thinking: Beyond STEM and arts integration. The STEAM Journal, 3(1), Article 11. https://doi.org/10.5642/ steam.20170301.11

Herrero, M. (Ed.). (2014a). Educación plástica 3. Editorial Santillana.

Herrero, M. (Ed.). (2014b). Educación plástica 6. Editorial Santillana.

Ibáñez-Ibáñez, M. M., Romero-López, M. D. C., y Jiménez-Tejada, M. D. P. (2019). ¿Qué ciencia se presenta en los libros de texto de Educación Secundaria? Enseñanza de las Ciencias, 37(3), 49-71. https://doi.org/10.5565/rev/ensciencias.2668 
Kim, Y., y Park, N. (2012). Development and application of STEAM teaching model based on the Rube Goldberg's invention. En S.-S. Yeo, Y. Pan, Y. S. Lee, y H. B. Chang (Eds.), Computer science and its applications: CSA 2012. (pp. 693-698). Springer. https://doi. org/10.1007/978-94-007-5699-1_70

Land, M. H. (2013). Full STEAM ahead: The benefits of integrating the arts into STEM. Procedia Computer Science, 20, 547-552. https://doi.org/10.1016/j.procs.2013.09.317

Liao, C. (2016). From interdisciplinary to transdisciplinary: An arts-integrated approach to STEAM education. Art Education, 69(6), 44-49. https://doi.org/10.1080/00043125.201 6.1224873

Ludeña, E. S. (2019). La educación STEAM y la cultura maker. Revista Padres y Maestros, 379, 45-51. https://doi.org/10.14422/pym.i379.y2019.008

McKeown, A. (2019). From STEM to STEAM at the beautiful midden field school: An artist/educator perspective. En A. de la Garza y C. Travis (Eds.), The STEAM revolution: Transdisciplinary approaches to science, technology, engineering, arts, humanities and mathematics (pp. 107-124). Springer. https://doi.org/10.1007/978-3-319-89818-6_8

Mengual, E., Gorgorió, N., y Albarracín, L. (2017). Análisis de las actividades propuestas por un libro de texto: el caso de la medida. REDIMAT Journal of Research in Mathematics education, 6(2), 136-163. https://doi.org/10.17583/redimat.2017.2415

Ministerio de Educación, Cultura y Deporte. (2014). Real Decreto 126/2014, de 28 de febrero, por el que se establece el currículo básico de la Educación Primaria. Boletín Oficial del Estado, 1 de marzo de 2014, (52), 19349-19420.

Peppler, K., y Wohlwend, K. (2018). Theorizing the nexus of STEAM practice. Arts Education Policy Review, 119(2), 88-99. https://doi.org/10.1080/10632913.2017.1316331

Portaankorva-Koivisto, P., y Havinga, M. (2019). Integrative phenomena in visual arts and mathematics. Journal of Mathematics and the Arts, 13(1-2), 4-24. https://doi.org/10. 1080/17513472.2018.1504269

Pro, C., y Pro, A. (2011). ¿Qué estamos enseñando con los libros de texto? La electricidad y la electrónica de tecnología en 3ํㅡㄹㅇ. Revista Eureka sobre Enseñanza y Divulgación de las Ciencias, 8(2), 149-170.

Quigley, C. F., y Herro, D. (2016). "Finding the joy in the unknown": Implementation of STEAM teaching practices in middle school science and math classrooms. Journal of Science Education and Technology, 25(3), 410-426. https://doi.org/10.1007/s10956016-9602-z

Quigley, C. F., Herro, D., King, E., y Plank, H. (2020). STEAM designed and enacted: Understanding the process of design and implementation of STEAM curriculum in an elementary school. Journal of Science Education Technology, 29(4), 499-518. https:// doi.org/10.1007/S10956-020-09832-W 
Rodríguez-Taboada, J., y García-Agra, P. (2019). Las matemáticas del arte. La Catarata.

Roel, V. G., Blanco, T. F., Framiñán, R. V., y Diego-Mantecón, J. M. (2019). Light and Geometry: An Artistic-Mathematical Experience in Primary School. In Bridges 2019 Conference Proceedings (pp. 427-430). Tessellations Publishing.

Rolling, J. H. (2016). Reinventing the STEAM engine for art + design education [Editorial]. Art Education, 69(4), 4-7. https://doi.org/10.1080/00043125.2016.1176848

Santaolalla, E., Gallego, D. J., y Urosa, B. (2015). Los libros de texto de matemáticas y su capacidad para desarrollar los distintos estilos de aprendizaje: estudio piloto. Revista de Estilos de Aprendizaje, 8(16), 178-210.

Sayers, J., Petersson, J., Rosenqvist, E., y Andrews, P. (2021). Opportunities to learn foundational number sense in three Swedish year one textbooks: Implications for the importation of overseas-authored materials. International Journal of Mathematical Education in Science and Technology, 52(4), 506-526. https://doi.org/10.1080/00207 39X.2019.1688406

Schubring, G., y Fan, L. (2018). Recent advances in mathematics textbook research and development: An overview. ZDM Mathematics Education, 50(5), 765-771. https://doi. org/10.1007/s11858-018-0979-4

Thibaut, L., Knipprath, H., Dehaene, W., y Depaepe, F. (2018). The influence of teachers' attitudes and school context on instructional practices in integrated STEM education. Teaching and Teacher Education, 71, 190-205. https://doi.org/10.1016/j.tate.2017.12.014

Thompson, D. R., y Senk, S. L. (2014). The same geometry textbook does not mean the same classroom enactment. ZDM-The International Journal on Mathematics Education, 46(5), 781-795. https://doi.org/10.1007/s11858-014-0622-y

Wang, J., y Lu, X. (2018). Selection of content in high school mathematics textbooks: An international comparison. ZDM Mathematics Education, 50(5), 813-826. https://doi. org/10.1007/s11858-018-0977-6

Wynn, T., y Harris, J. (2012). Toward a STEM + arts curriculum: Creating the teacher team. Art Education, 65(5), 42-47. https://doi.org/10.1080/00043125.2012.11519191

Zhbanova, K. S. (2019). Editorial: Developing creativity through STEM subjects integrated with the arts. Journal of STEM Arts, Crafts, and Constructions, 4(1), 1-15.

TERESA F. BLANCO

Dirección: Área de Didáctica de la Matemática. Facultad de Ciencias de la Educación. Universidad de Santiago de Compostela.

Av. de Xoán XXIII, 15782, A Coruña teref.blanco@usc.es

Teléfono: $\quad 881812012$ 